\title{
A STUDY ON PRESCRIBING PATTERN OF ANTIMICROBIAL AGENTS IN THE NEONATAL INTENSIVE CARE UNIT OF A TERTIARY CARE TEACHING HOSPITAL IN PUDUCHERRY, SOUTH INDIA
}

\author{
PANDIAMUNIAN J ${ }^{*}$, KARTIK J SALWE ${ }^{2}$, SOMASUNDARAM G ${ }^{3}$, BHANU PRAKASH KOLASANI ${ }^{1}$ \\ ${ }^{1}$ Department of Pharmacology, Vinayaka Mission's Medical College and Hospital, Vinayaka Mission's University, Karaikal, Puducherry, \\ India. ${ }^{2}$ Department of Pharmacology, Mahatma Gandhi Medical College and Research Institute, Sri Balaji Vidyapeeth, Puducherry, India. \\ ${ }^{3}$ Department of Pharmacology, Sri Lakshmi Narayana Institute of Medical Sciences, Puducherry, India. Email: jpandiamunian@gmail.com \\ Received: 05 June 2016, Revised and Accepted: 08 June 2016
}

\section{ABSTRACT}

Objective: This study was done to evaluate the current prescribing and usage pattern of antimicrobial agents (AMAs) in the neonatal intensive care unit (NICU).

Methods: A prospective, hospital-based, cross-sectional study (prescription audit) was carried out between May and August 2012 in the NICU of a Teaching Hospital. Data were collected by reviewing case records of all neonates admitted to the NICU during the study period.

Results: A total of 120 case records were reviewed and 100 were eligible to be included in the study. Out of the 100, no antimicrobial prescription was made in 35 case records. Remaining $65(65 \%)$ were prescribed at least one AMA. 167 AMA prescriptions were made in total and average number of AMAs utilized per neonate was 1.670. Cefotaxime (24.6\%) was the most commonly prescribed AMA followed by amoxicillin (23.4\%). Aminoglycosides (43.7\%) were the commonly prescribed group of AMAs followed by penicillins (28.7) and cephalosporins (24.6\%). Most of the AMAs were prescribed to non-bacteriologically proven infections (50.5\%). Among the 167 AMA prescriptions, 104 (62.28\%) were made in their brand names and 63(37.72\%) were made in their generic names.

Discussion and Conclusion: This study highlights current usage of AMAs in the NICU of a tertiary care teaching hospital in Puducherry. AMAs were found to be prescribed frequently without bacteriological evidence and in their brand names. This necessitates the need for motivating doctors to improve AMA prescriptions with supportive bacteriological evidence and in generic names.

Keywords: Antimicrobial agents, Neonatal intensive care unit, Generic drugs, Non-bacteriologically proven infections.

(c) 2016 The Authors. Published by Innovare Academic Sciences Pvt Ltd. This is an open access article under the CC BY license (http://creativecommons. org/licenses/by/4. 0/) DOI: http://dx.doi.org/10.22159/ajpcr.2016.v9i5.13277

\section{INTRODUCTION}

Each year in India, over 1 million newborns die before they complete their first month of life, accounting for $30 \%$ of the world's neonatal deaths [1]. Two-thirds of infant deaths in India occur in the first month of life (with one-third of all neonatal deaths associated with low birth weight) [2]. Newborn babies, who need intensive medical attention, are often admitted into a special area of the hospital called the Neonatal Intensive Care Unit (NICU). Most babies, admitted to the NICU are premature (born before 37 weeks of pregnancy), have low birth weight or have a medical condition that requires special care.

Globally, infection (36\%) is the most common cause of mortality in neonates followed by preterm birth (28\%) and birth asphyxia (23\%) [3-5]. In India, prematurity, sepsis, and birth asphyxia contribute to $85 \%$ of neonatal mortality [6]. All babies having an infection and those who are suspected to be having infection will receive AMAs in the NICU. All low birth weight infants admitted to a NICU also receive an empirical antibiotic treatment in the initial postnatal days, in spite of sterile cultures and low incidence of culture-proven bacterial sepsis in this population.

Several authors have reported concern about the continuous indiscriminate and excessive use of antimicrobial agents (AMAs) that promote the emergence of antibiotic-resistant organisms $[7,8]$. Prescribing drugs is an important skill which needs to be continuously assessed and refined accordingly. It not only reflects the physician's knowledge of pharmacology and pathophysiology but also his/her skill in diagnosis and selection of the most appropriate cost-effective treatment [9].
The study of prescribing patterns helps to monitor, evaluate and suggest alterations in practitioners' prescribing habits so as to make medical care rational and cost-effective. Hence, the present study was done to evaluate the prescribing pattern of AMAs to the patients who were admitted to the NICU of a tertiary care hospital in Puducherry.

\section{Objectives}

To evaluate the prescribing pattern of AMAs in the NICU of a tertiary care teaching hospital located in Puducherry.

\section{METHODS}

A prospective, hospital-based, cross-sectional study (prescription audit) was conducted in the NICU of a tertiary care rural hospital. This study was carried out between May 2012 and August 2012. Considering the total number of admitted neonates in the NICU in the previous 3 years under the department of pediatrics, 120 neonatal case records were recruited and reviewed for the present study (expecting $20 \%$ non-response and calculated sample size of 96 ). The study was carried out by the department of pharmacology in-collaboration with the Department Of Pediatrics.

\section{Ethical clearance}

Approval for the study was obtained from the Institutional Human Ethics Committee before the commencement of the study. Since the study did not involve any active intervention or participation of subjects, waiver for written informed consent was obtained from the institutional ethical committee. Subject confidentiality was maintained during and after data collection. 


\section{Exclusion criteria}

1. Incomplete patient case sheet

2. Discharged within $24 \mathrm{hrs}$ of admission

3. Transferred to other specialty intensive care units

4. Death of patient before hospital discharge.

\section{Data collection and procedure}

Data were collected through review of case records of neonates admitted and treated in the NICU of pediatrics department. Consecutive 120 case records of neonates admitted and treated in the NICU between May and August 2012 were obtained from the medical records department of the hospital. To evaluate the drug prescribing pattern, a data collection pro forma sheet was prepared. The pro forma contained space for recording the following data which included.

a. Demographic data: Except name, other demographic data including age and sex of the baby and address were noted in the pro forma.

b. Provisional and final diagnoses.

c. Duration of stay in the NICU.

d. Group and type of antimicrobials prescribed: Different groups and types of AMAs prescribed in the case records were noted down in detail.

e. Dosage, durations, and frequency: Dose of all AMAs, their duration of prescription and frequency were recorded.

f. Route of administration: Details regarding the route and formulation of administration were noted.

g. Brand or generic drugs: Whether generic or brand names of the drugs were mentioned in the case records was noted.

h. Any adverse reaction to the antimicrobials as noted by the physicians in the case records.

i. Investigation results: Details of culture and sensitivity, commonly isolated organisms and their resistance pattern were analyzed from the reports attached to the case records. Details of other markers of infection (C-reactive protein, organisms/pus cells in urine, detection of vegetation in ECHO, relevant radiologic findings (Ultrasonography, X-ray, CT).

Drugs other than AMAs, fluids and electrolyte solutions, amino acids, glucose, oxygen, phototherapy and prophylactic ophthalmic treatment were not considered in data collection.

\section{Categorization of AMA usage based on their indications}

Antimicrobial therapy was categorized according to the indication for the antimicrobial use. Four antimicrobial usage groups of subjects were essentially defined according to the indication on which the antimicrobial drugs were prescribed to the neonates.

- Usage Group 1: Bacteriologically proven infection was considered as the indication for antimicrobial prescription if culture and sensitivity report suggested organism growth and their drug sensitivity.

- Usage Group 2: Non-bacteriologically proven infection/empirical was considered as the indication for antimicrobial prescriptions if investigation data such as urine routine and radiological findings, and clinical findings were suggestive of local or systemic infection but the causative organism and its sensitivity pattern were unknown.

- Usage Group 3: The antimicrobial therapy was considered as prophylactic if there was no evidence of infections and the agent was employed to prevent infection (e.g., in catheterized patients).

- Usage Group 4: Indication was considered as symptomatic if the records suggested that AMAs were prescribed based on the clinical symptoms and signs (e.g., treatment of fever in the absence of specifically suspected infection).

\section{Statistical analysis}

Collected data were entered in Microsoft_Office_Excel 2010 and analyzed using SPSS Inc. Statistical Software Version 17.0. Descriptive statistical analysis was done using proportion, percentages and mean \pm standard deviation.

\section{RESULTS}

Out of the 120 records reviewed, 100 were eligible to be included in the study. Out of the 100, 54 (54\%) were male babies and 46 (46\%) were female babies. 9 (9\%) babies were from Puducherry and 91 (91\%) babies were from Tamil Nadu.

The most common diagnostic categories encountered were low birth weight (n-28), preterm (n-25), exaggerated physiological Jaundice (n-21), early onset neonatal sepsis (n-11), respiratory distress syndrome (n-9), intra uterine growth retardation (n-7), late onset neonatal sepsis (n-7), hydronephrosis (n-6), congenital heart disease (n-3), umbilical sepsis (n-2), pneumonia (n-3), and meconium aspiration syndrome (n-2)

Minimum duration of stay in the NICU in the study sample was 3 days and the maximum duration of stay was 17 days, and mean $( \pm \mathrm{SD})$ duration of stay was found to be $7.87( \pm 2.58)$ days. Out of the 100 cases records $35(35 \%)$ were not prescribed any antibiotics during their course of treatment. Remaining 65 (65\%) were prescribed at least one AMA (Fig. 1).

\section{Prescribing frequency of AMAs in the NICU}

About 167 antibiotic prescriptions were made for the 65 babies who received AMAs. Eight different types of AMAs were utilized. (Fixed drug combinations such as imipenem + cilastatin and piperacillin + tazobactam prescriptions were considered as a single type of AMA for analysis). Cefotaxime (24.6\%), ampicillin (23.4\%), amikacin $(23.4 \%)$, and gentamicin $(20.4 \%)$ were the most frequently prescribed AMAs in the NICU (Table 1). As depicted in Table 2, aminoglycosides (43.7\%), penicillins (28.7\%), and cephalosporins $(24.6 \%)$ were the predominantly utilized group of AMAs in the NICU. The majority of the admitted neonates received 2 to 4 numbers of AMAs during their course of treatment in the NICU as shown in the Fig. 2.

\section{Indications for AMA use in the NICU}

About 167 prescriptions made for the 65 babies were categorized into four usage groups, and the percentage of prescriptions based on usage was as shown in Table 3. AMAs were prescribed more frequently for non-bacteriologically proven infections (50.5\%) followed by for symptomatic indication (33.3\%). Only $11.8 \%$ of the antimicrobial usage was found to be based on the sensitivity pattern of the infective agents (Table 3).

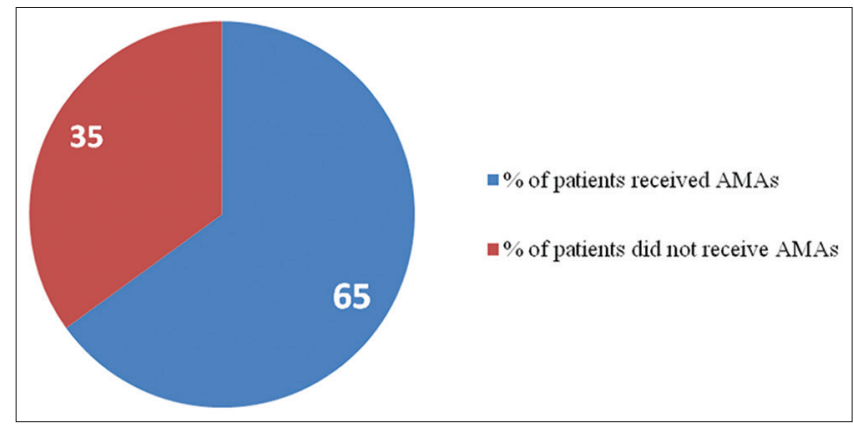

Fig. 1: Percentage of neonatal in-patients who received antimicrobial agents in the neonatal intensive care unit

Table 1: Prescribing frequency of systemic antimicrobial agents in the NICU

\begin{tabular}{lll}
\hline S. No & Drug & Frequency (\%) \\
\hline 1 & Cefotaxime & $41(24.6)$ \\
2 & Ampicillin & $39(23.4)$ \\
3 & Amikacin & $39(23.4)$ \\
4 & Gentamicin & $34(20.4)$ \\
5 & Piperacillin+tazobactam & $7(4.2)$ \\
6 & Metronidazole & $3(1.8)$ \\
7 & Imipenem+cilastatin & $2(1.2)$ \\
8 & Cloxacillin & $2(1.2)$ \\
& Total & $167(100)$ \\
\hline
\end{tabular}

NICU: Neonatal intensive care unit 


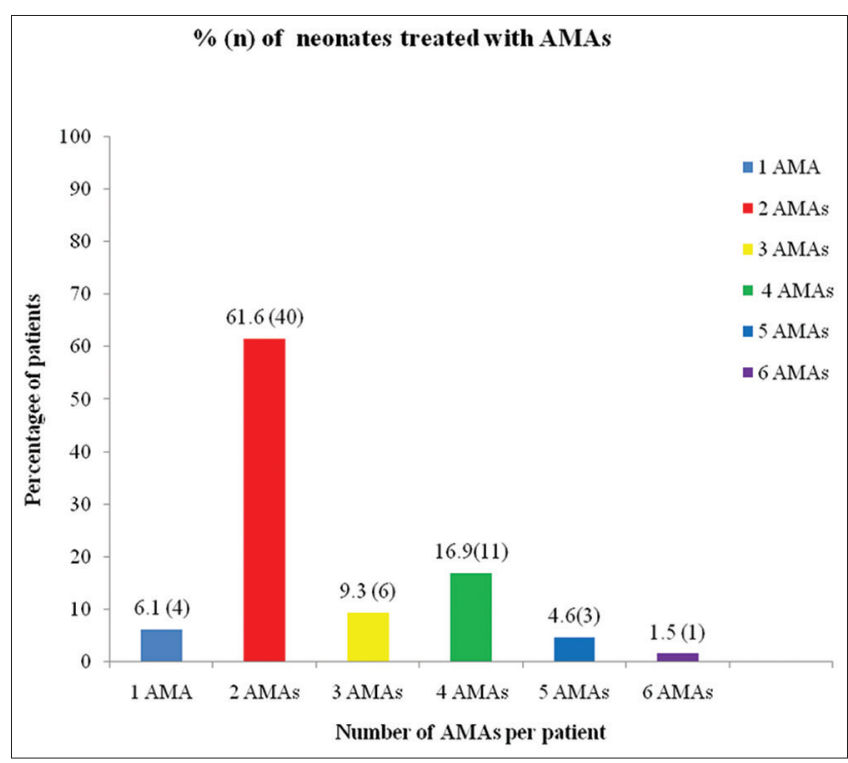

Fig. 2: Distribution of the neonates (treated with antimicrobial agents) based on the number of antimicrobial agents prescribed to them

Table 2: Utilization of various groups of AMAs in the NICU

\begin{tabular}{lll}
\hline S. No & Group of AMAs & AMA prescriptions N (\%) \\
\hline 1 & Aminoglycosides & $73(43.7)$ \\
2 & Penicillins & $48(28.7)$ \\
3 & Cephalosporins & $41(24.6)$ \\
4 & Amebicides & $3(1.8)$ \\
5 & Carbapenems & $2(1.2)$ \\
& Total & $167(100)$ \\
\hline
\end{tabular}

AMAs: Antimicrobial agents, NICU: Neonatal intensive care unit

Table 3: Indication for AMA usage in the NICU

\begin{tabular}{lll}
\hline S. No & Usage group & Percentage \\
\hline 1 & $\begin{array}{l}\text { Usage Group 1: Bacteriologically } \\
\text { proven infection }\end{array}$ & 11.8 \\
& $\begin{array}{l}\text { Usage Group 2: Non-bacteriologically } \\
\text { proven infection }\end{array}$ & 50.5 \\
& Usage Group 3: Prophylaxis & 4.4 \\
3 & Usage Group 4: Symptomatic & 33.3 \\
\hline \multicolumn{2}{l}{ AMAs: Antimicrobial agents, NICU: Neonatal intensive care unit }
\end{tabular}

Routes of administration of AMAs

All the 167 AMA prescriptions in the NICU were administered in injection form parenterally (intravenously [IV]).

\section{Culture and sensitivity reports}

Blood samples were sent for culture and sensitivity testing from 44 in-patients. Out of these, seven blood culture samples were positive for bacterial growth (coagulase negative-Staphylococci - 4 times; nonfermentum organisms - twice and klebsiella - once). All these were predominantly sensitive to AMAs such as cefotaxime, amoxicillin, amikacin and gentamicin. In one of the culture report, Staphylococcus was found to be sensitive to vancomycin, linezolid, and tetracycline; resistant to methicillin.

\section{Percentage of AMAs prescribed in the generic name}

Among the 167 AMAs prescribed, 63 (37.72\%) were prescribed in generic names and the remaining $104(62.28 \%)$ were prescribed in brand names. No adverse reaction to any prescribed AMA was noticed in the case records.

\section{DISCUSSION}

The study aimed at analyzing the prescribing pattern of AMAs in the NICU of a tertiary care teaching hospital. Male preponderance (54\%) in admissions was noticed in the study. Mean duration of stay of neonates in the NICU was $7.87 \pm 2.58$ days. Low birth weight, preterm and exaggerated physiological jaundice were the most frequently encountered diagnoses which necessitated admission of neonates to the NICU. $35 \%$ of the neonatal admissions did not receive any AMAs during their course of treatment. $65 \%$ of them were treated with at least one AMA. This is lesser than a German study finding which reported that $90.7 \%$ of neonatal patients were prescribed at least one AMA in NICU. Mostly, two AMAs were used for a patient, which is in accordance with the German study [10]. On an average 1.67 number of AMAs were prescribed per each neonate, which is lesser than the finding (3.4 AMAs/patient) of Natalie Schellack and Gous study done in South Africa [11]. The IV route (100\%) was found to be the only route of drug administration in the studied NICU, whereas a little lesser proportion (92.1\% of AMAs given IV) was given parenterally in a study done in Eastern India [12]

Eight different types of AMAs were prescribed for neonates in the NICU. Cefotaxime, amikacin, ampicillin and gentamicin were the most frequently prescribed AMAs in the NICU, which altogether constituted 91.6\% of AMA prescriptions. Ampicillin was found to be frequently coprescribed with gentamicin and cefotaxime with amikacin. The findings are comparable with other similar study findings that cefotaxime (a third generation cephalosporin), ampicillin and gentamicin were the most frequently utilized AMAs in the NICUs [12-14]. In accordance with this study finding, the drugs belonging to the cephalosporins were found to be the predominantly prescribed AMAs in many of the European hospitals [15-18].

Aminoglycoside had been the most frequently (43.7\%) utilized group of antimicrobials in the studied NICU. In combination with either a cephalosporin or penicillin, one of the aminoglycosides were prescribed to the majority of the neonates who received AMAs in the NICU. This is in accordance with other study reports, wherein gentamicin was found to be widely used in combination with $\beta$-lactam antibiotics, especially crystalline penicillin and ampicillin as this combination will provide synergistic activity against the most common pathogens isolated in early onset sepsis (e.g., Klebsiella pneumoniae and coagulase-negative Staphylococci) [19-21]

AMAs were found to be prescribed mostly (50.5\%) on the basis of non-bacteriologically proven infections followed by symptomatic basis $(33.3 \%)$. This shows that majority of the times it was not possible for the clinicians to detect the exact causative organism and their sensitivity pattern.

Only $37.72 \%$ of the utilized AMAs were prescribed in generic names, and $62.28 \%$ of antimicrobial prescriptions were made in brand names. This may increase the financial burden of patient families. Measures to improve prescribing drugs in generic names need to be implemented.

\section{CONCLUSION}

Based on the results, we conclude that cefotaxime and aminoglycosides, respectively, were the most frequently prescribed AMA and Group in the NICU studied. Only a small proportion $(11.8 \%)$ of the AMA prescription was made based on bacteriological culture and sensitivity evidence. It was also noted in the study that culture and sensitivity testing was not done in the majority of the neonates who were treated with AMAs. Most $(88.2 \%)$ of the AMA prescriptions were made based on clinical diagnosis, other investigation reports and the physician's experiences based on symptoms. Efforts need to be undertaken for increasing the proportion of AMA prescriptions for bacteriologically proven infections based on their culture and sensitivity evidence. This will help in prevention of drug resistance to the frequently prescribed AMAs. 
It is being evident from the study result that AMAs are prescribed predominantly in brand names in the NICU. Measures need to be undertaken to encourage physicians to prescribe AMAs in generic names to minimize health care cost.

\section{REFERENCES}

1. USAID. Maternal and Child Health - 2004. USAID Battles Neonatal Deaths in India. 2005. Available from: http://www.usaid.gov/our_work/ global_health/home/News/ghachievements.html.

2. Yasmin S, Osrin D, Paul E, Costello A. Neonatal mortality of low-birth-weight infants in Bangladesh. Bull World Health Organ 2001;79(7):608-14.

3. World Health Report 2005: Make Every Mother and Child Count. Geneva: WHO; 2005

4. Lawn JE, Cousens S, Zupan J; Lancet Neonatal Survival Steering Team. 4 million neonatal deaths: When? Where? Why? Lancet 2005;365(9462):891-900.

5. Lawn JE, Cousens SN, Wilczynska K. Estimating the causes of four million neonatal deaths in the year 2000: Statistical annex. In: The World Health Report 2005. Geneva: WHO; 2005.

6. Bang AT, Paul VK, Reddy HM, Baitule SB. Why do neonates die in rural Gadchiroli, India? (Part I): Primary causes of death assigned by neonatologist based on prospectively observed records. J Perinatol 2005;25 Suppl 1:S29-34.

7. Niederman MS. Appropriate use of antimicrobial agents: Challenges and strategies for improvement. Crit Care Med 2003;31(2):608-16.

8. Pulcini C, Pradier C, Samat-Long C, Hyvernat H, Bernardin G, Ichai C, et al. Factors associated with adherence to infectious diseases advice in two intensive care units. J Antimicrob Chemother 2006;57(3):546-50.

9. Benet LZ. Principles of prescription order writing and patients compliance instructions. In: Goodman AG, Rall TW, Nies AS, Taylor P, editors. Goodman and Gilman's The Pharmacological Basis of
Therapeutics. $8^{\text {th }}$ ed. New York: Pergamon Press Inc.; 1991. p. 1640.

10. Neubert A, Lukas K, Leis T, Dormann H, Brune K, Rascher W. Drug utilisation on a preterm and neonatal intensive care unit in Germany: A prospective, cohort-based analysis. Eur J Clin Pharmacol 2010;66(1):87-95

11. Schellack N, Gous AG. Antibiotic prescribing patterns in a neonata intensive care unit. South Afr J Epidemiol Infect 2011;26(4):267-70.

12. Chatterjee S, Mandal A, Lyle N, Mukherjee S, Singh AK. Drug utilization study in a neonatology unit of a tertiary care hospital in eastern India. Pharmacoepidemiol Drug Saf 2007;16(10):1141-5.

13. Warrier I, Du W, Natarajan G, Salari V, Aranda J. Patterns of drug utilization in a neonatal intensive care unit. J Clin Pharmacol 2006;46(4):449-55

14. Fanos V, Cuzzolin L, Atzei A, Testa M. Antibiotics and antifungals in neonatal intensive care units: A review. J Chemother 2007;19(1):5-20.

15. ESAC Year Book; 2008. Available from: http://www.esac.ua.ac.be

16. Dimina E, Akermanis M, Dumpis U. Antibiotic consumption in latvian teaching hospital 2000-2008. Proc Latv Acad Sci 2009;63:253-6.

17. Borg AM, Zarb P. Consumption of antibiotics at St Luke's hospital Malta Med J 2006;18(1):33-8.

18. Brusic-Renaud J, AntunovicM, Subjagic V. The analysis of antibiotic consumption within the tertiary healthcare institution in Serbia during 10-year period (2001-2010). Int J Pharm Pharm Sci 2016;8(5):401-3.

19. Fullas F, Padomek MT, Thieman CJ, Van Gorp AE. Comparative evaluation of six extended-interval gentamicin dosing regimens in premature and full-term neonates. Am J Health Syst Pharm 2011;68(1):52-6.

20. Chattopadhyay B. Newborns and gentamicin - how much and how often? J Antimicrob Chemothe 2002;49(1):13-6

21. Begg EJ, Vella-Brincat JW, Robertshawe B, McMurtrie MJ Kirkpatrick CM, Darlow B. Eight years' experience of an extendedinterval dosing protocol for gentamicin in neonates. J Antimicrob Chemothe 2009;63(5):1043-9 